\title{
Localization and quantification of dystrophin in epileptic rats
}

\author{
W.T.E. Yeung \\ Maastricht university department of neuroscience \\ e.yeung@student.maastrichtuniversity.nl
}

\section{Abstract}

Aim: In Duchenne muscular dystrophy (DMD) populations, a higher prevalence of epilepsy has been found compared with control groups. We hypothesized that epilepsy/seizures causes loss of dystrophin in the brain. Our aim was to localize dystrophin in cerebellum and hippocampus and to develop a reliable method for quantification of dystrophin. Methods: Male adult Spraque-Dawley rats were used in a amygdala kindled model $(\mathrm{N}=24)$. Cerebellar and hippocampal tissue were stained immunohistochemically with anti-dystrophin, anti-calbindin, anti-GFAP and Hoechst. Anti-NeuN was only applied in hippocampal tissue. Under fluorescent microscope, pictures were taken for quantification in ImageJ. Results: Glia cells and Purkinje cells colocalized with dystrophin, however, hippocampal neuronal cells did not show a colocalization with dystrophin. Statistical analysis of the quantification of dystrophin around the Purkinje neuron, showed a high intra-observer correlation (Mean grey value: Pearson correlation $(r=1,000, P<0,0001)$ and ICC ( $r=1,000, \mathrm{P}<0,0001)$; Intensity density: Pearson correlation $(r=0,986, \mathrm{P}<0,000)$ and ICC $(r=0,984, P<0,0001))$. Conclusion: Dystrophin is ubiquitously expressed in the cerebellum and hippocampus, but the exact distribution of the dystrophin isoforms in these areas are not clear yet. We have developed a reliable quantification method of dystrophin around the cerebellar Purkinje cells, but not for glia cells and hippocampal neurons. Future studies should therefore not only be aimed at the distribution of the dystrophin isoforms, but also at the quantification of glia cells and hippocampal neurons.

\section{Keywords}

Dystrophin, Duchenne, colocalization, epilepsy, quantification. 


\section{Introduction}

Duchenne muscular dystrophy (DMD) is a X chromosome-linked recessive neuromuscular disorder affecting approximately 1 in every 3500 alive male newborns. The disorder is caused by a mutation in the dystrophin gene, which results in progressive muscle degeneration caused by the replacement of muscle tissue with fat and fibrous tissue occurring not only in skeletal muscles, but also in heart, respiratory muscles and in the central nervous system (CNS). Degeneration in muscles causes significant motor development disorder and muscle weakness, starting in the legs and pelvis. In the brain, degeneration causes cognitive and behavioural disabilities.

Dystrophin is a cytoplasmic protein linking cytoskeletal actin filaments to membrane proteins that is abundantly expressed in muscular tissue, but it can also be found in the retina, kidney and CNS. Therefore, some DMD patients have less dystrophin in the brain, depending on the location of the mutation.

Dystrophin is involved in the clustering of different ion channels and postsynaptic membrane receptors in the brain, for example the clustering of aquaporin channels, kainate-type glutamate receptors and particularly $G A B A_{A}$ receptors, which suggests that a loss of its function may be the cause of cognitive deficits in DMD patients.

Dystrophin exists of a few isoforms, these include Dp427, Dp 71 and Dp140. In the brain Dp427 is expressed in hippocampal neurons, cortical pyramidal cells, cerebellar Purkinje cells and Dp427 has been found to colocalize with $\gamma$-aminobutyric acid type A (GABA A receptors and seems to play an important role in the clustering and stabilization/ anchoring of $G A B A_{A}$ receptors. Dp71 in the brain is found in neurons and glia, most notably in perivascular astrocytes and cerebellar Bergman glia. Expressed the specialized endfeet processes of perivascular astrocytes, Dp71 is suggested to play a role in maintaining the function of the blood brain barrier (BBB), in which aquaporin 4 plays a role and Dp71 appears to be involved in the clustering of potassium channel Kir 4.1 that maintains potassium homestasis in astrocytes.

The prevalence of epilepsy is higher in DMD population compared in control population; Goodwin et al. reported a prevalence of 3,1\%, Etemadifar and Molaei 12,3\% and Pane et al. $6,3 \%$ in DMD population compared with control populations of $0,4 \%-0,5 \%, 0,5 \%-1 \%$ and $0,5 \%-1 \%$ respectively. These reports raised the question whether a reduced expression of dystrophin is linked with increased seizure susceptibility. 
The question remains if dystrophin alterations are cause or consequence of epileptogenesis/seizures, because the studies are based on patients who have been suffering from epilepsy for years and on animal models of epilepsy/seizures. Therefore, the aim of this study was to determine this question. We hypothized that the presence of seizures causes loss/decrease of dystrophin and that the decrease of dystrophin is a consequence of epileptogenesis in a rodent model for temporal lobe epilepsy. In this hypothesis, the aim was to localize dystrophin in the cerebellum and hippocampus and to produce a reliable method to quantify the dystrophin.

\section{Material and methods}

In the amygdala kindling model, a rodent model for temporal lobe epilepsy, immunohistochemistry stainings were performed on hippocampal and cerebellar tissuee for the expression of dystrophin. Male adult Spraque-Dawley rats were used. The rats could adapt to the laboratory for a week before the surgery. Surgical procedures took place under general isoflurane anaesthesia. For the implantation of the electrode, a standard rat sterotac was used. The electrode used, was a bipolar platinum/iridium needle, implanted in the left basolateral amygdala (coordinates relative to bregma $-2,5 \mathrm{~mm}$ posteriorly, 4,8 $\mathrm{mm}$ laterally and 9,6 $\mathrm{mm}$ ventrally). One week after surgery, stimulation of the amygdala took place twice a day $(400 \mu, 50 \mathrm{~Hz}, 0.2 \mathrm{~ms}$ blockpulses). The fully kindled stage was reached when the rats underwent five consecutive stage $V$ seizures according to Racine's grading. When this stage was reached, stimulation was reduced to once a day for two weeks. The rats were sacrificed and cerebellar and hippocampal tissue were used for immunohistochemistry.

\section{Pilot study 1}

The cerebellar tissue was split into two even groups to perform two different staining protocols: one for anti-dystrophin, anti-calbindin and Hoechst incubation and the other for anti-dystrophin, anti-GFAP and Hoechst incubation. The samples were washed and blocked for 60 minutes with blocking buffer to minimize non-specific absorption of the antibodies. After blocking, incubation with primary anti-dystrophin was performed overnight at $4{ }^{\circ} \mathrm{C}$ with a 1:500 dilution in blocking buffer. The samples were washed and incubated with secondary antibody diluted 1:200 in blocking buffer for 2 hours. After washing, the samples were either incubated with primary anti-calbindin in THST buffer or primary anti-GFAP in $0,1 \%$ Triton in PBS buffer overnight at $4{ }^{\circ} \mathrm{C}$. The samples were washed and incubated for 1 hour with secondary antibody with a 1:200 dilution in THST buffer. Samples were counterstained with Hoechst diluted 1:500 for 30 minutes after washing. 
Samples were washed and mounted on gelatin coated slides with $80 \%$ glycerol in TBS and closed with nail polish.

\section{Pilot study 2}

Hippocampal tissue of two amygdala kindled rats were stained anti-NeuN, anti-dystrophin and Hoechst. The samples were washed and blocked with blocking buffer for 60 minutes. After blocking, incubation with primary anti-dystrophin was performed overnight at $4{ }^{\circ} \mathrm{C}$ with a 1:500 dilution in blocking buffer. These samples were washed and incubated with secondary antibody diluted 1:200 in blocking buffer 1 for 2 hours. Following washing, incubation was performed with primary antibody NeuN diluted 1:1000 in 0,1\% Triton $\mathrm{X}-100$ and $3 \%$ NDS PBS dilution at $4{ }^{\circ} \mathrm{C}$ overnight. The samples were washed and incubated with secondary antibody diluted 1:200 in 4\% NDS diluted in 1X PBS. The samples were counterstained with Hoechst diluted 1:500 in PBS for 30 minutes after washing. Samples were washed and mounted on gelatin coated slides with $80 \%$ glycerol in TBS and closed with nail polish.

\section{Quantification of immunohistochemical signal}

After the stainings, pictures were taken using the software micromanager with fluorescent microscope BX51. Pictures of Purkinje cells and astrocytes were taken in the cerebellum, while in the hippocampus pictures of astrocytes and neuronal hippocampal cells of the different regions were taken under a magnification of 20, 40 and 100 times. For each region of interest, photos were made with different fluorescent channel; TXRED (red), FITC (green) and DAPI (blue) to visualize calbindin or GFAP (depends on the staining), dystrophin and cell nuclei respectively. Pictures of the Purkinje cells were used for quantification with the sofware ImageJ. First, the red and green pictures were merged to better visualize colocalization of dystrophin and calbindine. By hand, dystrophin around the Purkinje cell was delineated two times for each cell; around the inner and outer membrane and the area was saved in ROI manager. Afterwards, area, mean gray value and intensity density were measured only in the green picture to prevent measuring signals other than dystrophin.

\section{Statistical analysis}

In order to measure the reliability of this self-constructed quantification method, intraobserver correlation was calculated using Pearson's correlation and intra-class coefficient (ICC) in SPSS with 10 cells per group (i.e. stimulated and sham), thereby generating a total of 20 cells (based on a power analysis which revealed that the number of 20 cells would be sufficient). The mean grey value and the intensity density of the outside and inside of 
the Purkinje cell was measured. Subsequently, the substraction of these two values (inside and outside delineation of dystrophin surrounding the Purkinje cell) was used as a value for dystrophin expression around that specific Purkinje cell. Several days later the same procedure was performed in a different order. These two values were used to calculate the intra-observer correlation (Pearson correlation and intra-class correlation) of the mean grey value and the intensity density.

\section{Results}

\section{Immunofluorescence}

In the cerebellum, dystrophin was localized around the Purkinje somatic and dendritic membranes. Here, the same can be seen. When the dystrophin and calbindin pictures were merged, dystrophin is localized around the Purkinje cells. In some Purkinje cells yellow was seen to suggest colocalization, while some did not (figure 1). Negative control of primary dystrophin antibody showed no immunoreactivity in Purkinje neurons.

In the cerebellum sections stained with GFAP, an abundant amount of astrocytes can be seen and dystrophin seems to be expressed at the same places as GFAP (figure 2). When GFAP was merged with dystrophin, the astrocytes were yellow, which suggests, again, a profound colocalization of dystrophin and astrocytes in the cerebellum (figure $2 \mathrm{~A}, \mathrm{C}, \mathrm{F}$ ). This is a new finding, as other authors failed to demonstrate dystrophin in other structures than Purkinje cells. Interestingly, in both the dystrophin and GFAP staining, dystrophin and glia seem to surround a round structure (figure $2 B, D$ ). When both pictures were merged, these round structures were surrounded by yellow ellipses (figure $2 \mathrm{~F}$ ).

In the hippocampus, GFAP labelling also showed an abundant amount of astrocytes. When comparing the dystrophin and GFAP pictures, it seems that the structures seen on both pictures are in a similar locations (figure $3 A, B, C, D$ ). Indeed, when the pictures were merged, the astrocytes are yellow. So it would seem that dystrophin and astrocytes also colocalize in the hippocampus (figure $3 \mathrm{E}, \mathrm{F}$ ).

With NeuN labelling, which is standard used for hippocampal neuron stainings, it was difficult to distinguish single cells due to a high cell density. In the dystrophin pictures, dystrophin surrounds round/oval structures in which the cells labelled with NeuN seem to fit in. However, no dystrophin was seen around neuronal hippocampal cells, which suggests that there is no colocalization of dystrophin and neuronal cells (figure $4 \mathrm{E}, \mathrm{F}$ ). 
Statistical analysis

Table 1. Pearson correlation and ICC of the mean grey value and intensity density ${ }^{* *}$. Correlation is significant at the 0,01 level (2-tailed).

\begin{tabular}{lll} 
& Pearson correlation & ICC \\
\hline Mean grey value & $1,000 \times \times$ & 1,000 \\
\hline Intensity density & $0,986 \times \times$ & 0,984 \\
\hline
\end{tabular}

Because it was initially thought that no signal was present of neuronal cells in calbindin labelled hippocampal tissue, only dystrophin around the glia cells and around the Purkinje cell were quantified. However, for glia cells, the quantification method was not compatible, because the axons and dendrites were thin and irregular, so manually delineation was not possible. At the end only dystrophin around Purkinje cells in the cerebellum were quantified.

Pearson correlation and ICC of the mean grey value were both 1,000, which means a significant correlation $(\mathrm{P}<0,0001)$ (table 1). For the intensity density, Peason correlation was 0,986 and ICC was 0,984 ; both showed a significant correlation $(\mathrm{P}<0,0001)$ (table 1).

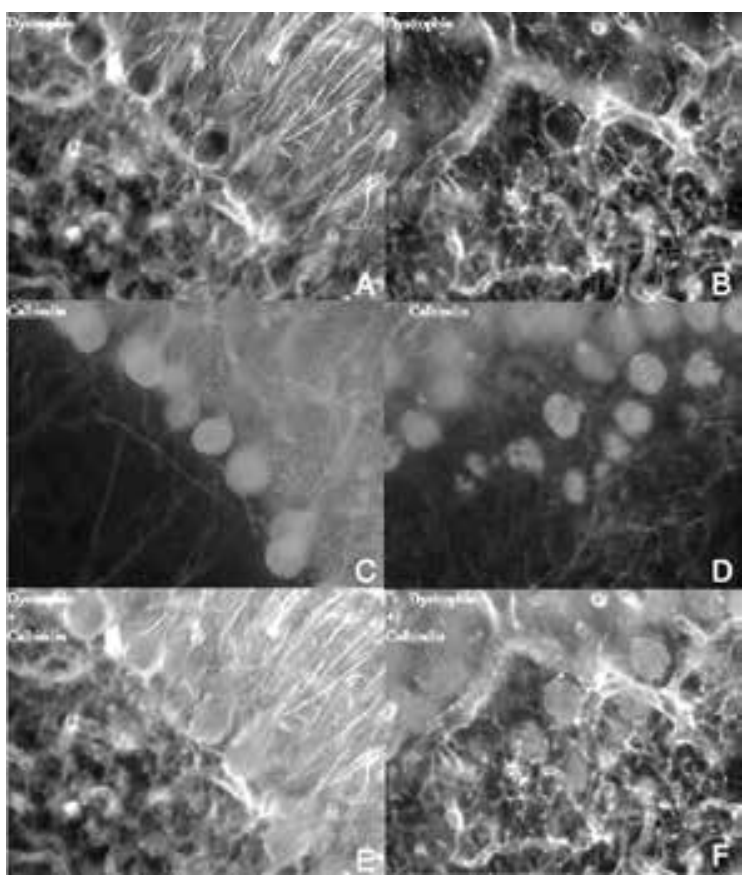

Figure 1. Single-channel and dualchannel images (40x) of Purkinje cells stained for dystrophin (green) and calbindin (red) in the cerebellum of amygdala-kindled rats. ${ }^{*}$ Note that the yellow color reflects colocalisation of dystrophin with cerebellar Purkinje cells, where calbindin is expressed. *Note these pictures are not from two conditions, but just typical examples 

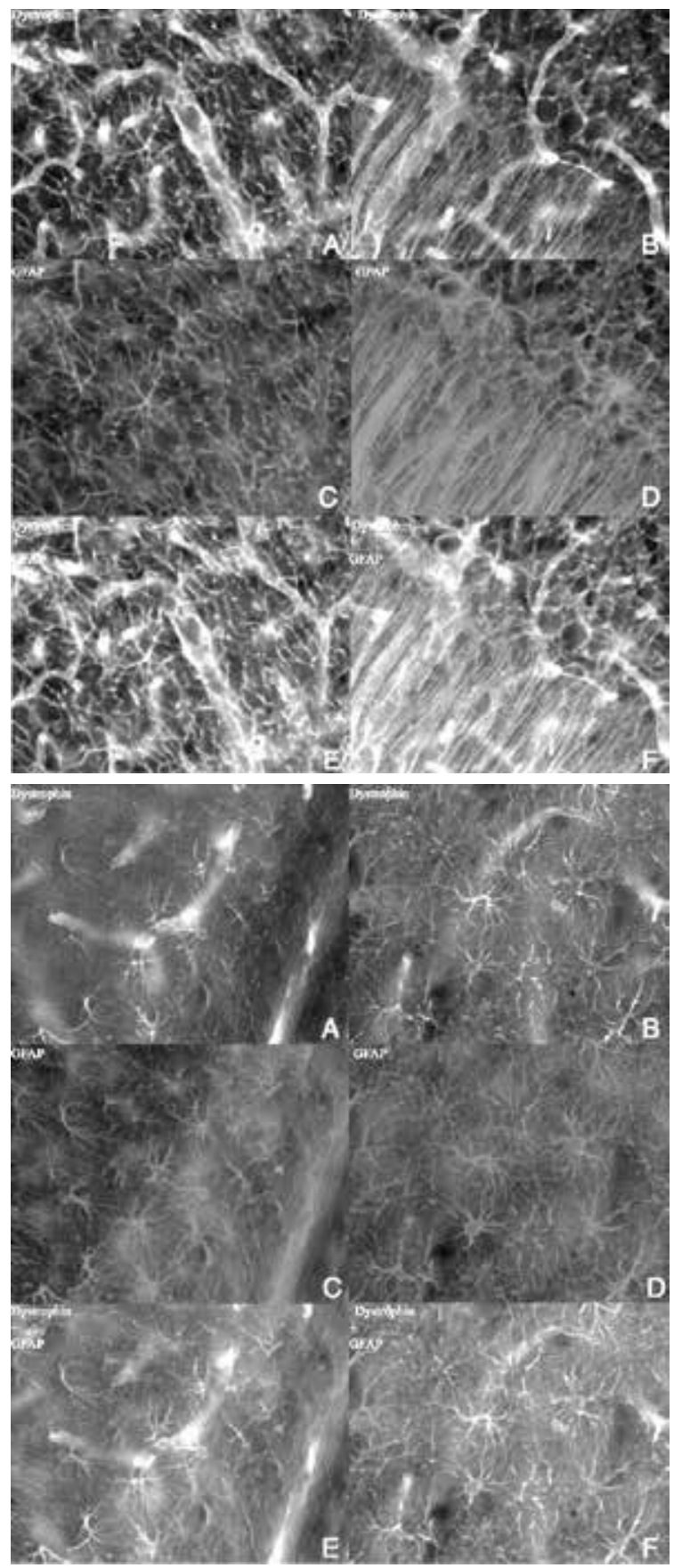

Figure 2. Single-channel and dualchannel images (40x) of glia cells stained for dystrophin (green) and GFAP (red) in the cerebellum of amygdala-kindled rats.

Figure 3. Single-channel and dualchannel images (40x) of glia cells stained for dystrophin (green) and GFAP (red) in the hippocampus of amygdala-kindled rats 


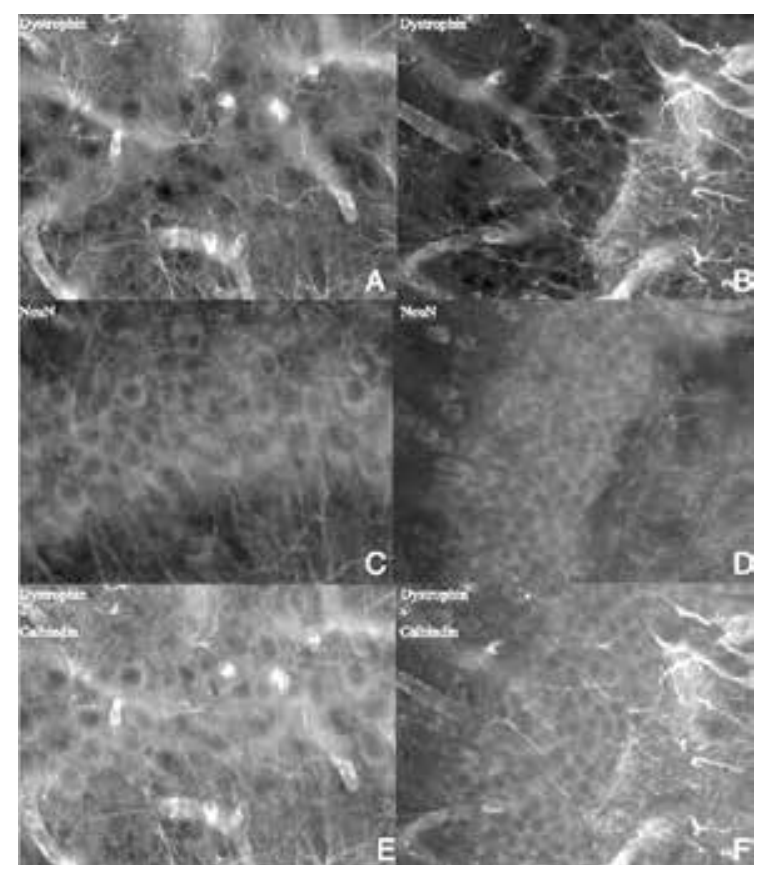

Figure 4. Single-channel and dual-channel images (40x) of hippocampal neuronal cells stained for dystrophin (green) and NeuN (red) in the hippocampus of amygdala-kindled rats.

\section{Discussion/Conclusion}

Dystrophin in the cerebellum

Double-labelling of calbindin and dystrophin allowed for examination of the localization of dystrophin in the cerebellum. In this study, it is confirmed that dystrophin is indeed expressed in the cerebellum and dystrophin seems to colocalize with Purkinje cells as well as with glia cells (figure 1, 2).

However, it is unknown in what isoforms dystrophin is distributed in Purkinje and glia cells. As was mentioned before, Dp427 and Dp71 are both expressed in neurons. Dp427 is only found in neurons in hippocampal cells, cortical pyramidal cells and cerebellar Purkinje cells, while Dp71 is found in glia cells and neurons. So the dystrophin found around the Purkinje cell, might consist of the isoforms Dp427 or Dp71, which could not be distinguished by the dystrophin antibody used in this study. Another problem is that this antibody binds to all isoforms since it is directed against the C-terminal of the dystrophin protein, thereby also staining Dp140. Since not much is known about Dp140, it cannot be excluded that Dp140 might be present in the stainings here as well. Further research is necessary to answer these questions. 
For a more specific staining, two antibodies against dystrophin could be used: one antibody directed against the $\mathrm{N}$-terminal of the protein, which binds to all isoforms and one antibody directed against the C-terminal, which is specific for the full-length Dp427 isoform. The difference of dystrophin stained by the two antibodies, is thus Dp140 or Dp71. For stainings against only one isoform, it might be possible to use existing monoclonal antibodies against these isoforms. In glia cells, a colocalization between GFAP-positive glia cells and dystrophin is suggested (figure 2), in contrast to previous literature. A few research groups, were not able to detect dystrophin immunofluorescence in structures or processes besides Purkinje cells in the cerebellum. However, these studies are over 20 years ago. We report here an obvious co-localization between astrocytes (GFAP) and dystrophin in the cerebellar cortex. The dystrophin colocalized with the glia cells could be Dp71 or Dp140; both are expressed in glia cells, Dp140 specifically in microvascular glia cells. To determine if Dp71 and Dp140 is expressed in the colocalized dystrophin, new antibodies have to be developed or generated as mentioned above, because the antibody used, binds to all isoforms.

Interestingly, dystrophin and glia seem to surround a round structure that looks like a Purkinje neuron (figure 2). This result, together with the finding that dystrophin is expressed in glia cells and does not fully colocalize, leads to a potential explanation for the dystrophin around the Purkinje cell, the Bergmann glia. Bergmann glia plays an important role in neuronal migration and contributes to the survival of Purkinje cells. Bergmann glia are expressed in the Purkinje cell layer and there is a possibility that the Bergmann glia surrounds the Purkinje cell with its processes. Therefore, the figures $(1,2)$ as shown before, could possibly display dystrophin localized in Bergmann glia cells and not in Purkinje cells.

The use of the marker calbindin in Purkinje neurons, could provide another explanation for the not fully colocalized dystrophin in Purkinje neurons. Calbindin is actually a cytoplasmic $\mathrm{Ca}^{2+}$ binding protein and thus does not bind to the membrane of the Purkinje neuron, while dystrophin is an anchoring protein found at the membrane. So it is possible that dystrophin does colocalize with Purkinje neuron at the membrane, which could not be visualized in this staining.

To investigate this possibility, triple staining could be done with differently generated primary antibodies. If it appears to be Bergman glia, for sure that isoform is Dp71. However, when it appears to be Purkinje cells where the dystrophin is located, no conclusion can be made as both Dp427 and Dp71 are expressed in neurons. If this is the case, staining against the $\mathrm{N}$-terminal and the $\mathrm{C}$-terminal of the protein could be used. 


\section{Dystrophin in the hippocampus}

With the NeuN labelling, the neuronal cells were not distinguishable. The cell density seems to be very high and the cells blurry. This could be caused by the use of a widefield fluorescence microscope, $\mathrm{BX}_{51}$, so more out-of-focus light is present. Also, no colocalization could be found between NeuN labelled neurons and dystrophin (figure 4). NeuN is a specific protein expressed in post-mitotic neurons and primarily stains the neuronal nucleus, but also cytoplasm and dendrites. However, dystrophin is found at the celmembrane and thus might still possibly colocalize with hippocampal neuronal cells at the membrane, which is not visible with the used staining.

In glia cells, a colocalization between GFAP-positive glia cells and dystrophin is suggested (figure 3), which is in contrast to previous literature. Here, it is also unknown which isotype is expressed in these cells.

\section{Quantification of dystrophin around Purkinje cells}

The intra-observer correlation of both the mean grey value and intensity density were very high for as well the Pearson correlation $(r=1,000, P<0,0001$ for mean grey value and $r=0,986, P<0,0001$ for the intensity density) as for the ICC ( $r=1,000, P<0,0001$ for mean grey value and $r=0,984, P<0,0001$ for the intensity density). A r-value close to 1 suggest a perfect correlation and thus suggests that the method seems reliable. An interobserver correlation calculation might be useful in the near future. It is expected that the interobserver correlation will be high, if the other person is consistent in his/her method. As was mentioned before, delineation of glia cells in the cerebellum and hippocampus was not possible manually, because the glia cells with the dystrophin have many thin dendrites, which could not be delineated accurately. Another method is necessary. A possible solution is to count the dystrophin positive glia cells and express these cells in a percentage for comparison between the experimental groups.

\section{Conclusion}

Dystrophin is ubiquitously expressed in the cerebellum and hippocampus, but the exact distribution of the dystrophin isoforms in these areas are not clear yet. We, however, demonstrate here to have developed an adequate protocol for dystrophin staining of the cerebellum and hippocampus. Also, we have developed a reliable manual quantification method of dystrophin around the cerebellar Purkinje cells. The more challenging part seems to be the quantification of dystrophin in cerebellar and hippocampal glia cells and hippocampal neurons. Future studies should therefore be aimed at tackling such problems. 
Although, manually quantification of dystrophin seems to be reliable, a few problems are still present, besides the fact that this is a time-consuming method. Therefore alternative methods in the area of semi-automated quantification should be sought. As a result, further research in different methods is necessary.

\section{Role of the student}

W.T.E. Yeung was an undergraduate student working under the supervision of Dr. G. Hoogland. The topic was proposed by PhD student R. Hendriksen. The student did one staining and the quantification by herself. The paper was written under guidance of PhD student R. Hendriksen and S. Schipper.

\section{References}

1. Li W., Zheng Y., Zhang W., Wang Z., Xiao J., Yuan Y. Progression and variation of fatty infiltration of the thigh mucles in Duchenne muscular dystropy, a muscle magnetic resonance imaging study. Neuromuscul Disord. 2015 May;25(5):375-80

2. van Ruiten H.J.A., Straub V., Bushby K., Guglieru M. Improving recognition of Duchenne muscular dystrophy: a retrospective case note review. Arch Dis Child. 2014;99:1074-1077

3. Elliot S.A., Davidson Z.E., Davies P.S., Truby H. A bedside measure of body composition in Duchenne muscular dystrophy. Pediatr Neurol. 2015 Jan;52(1):82-7

4. De Sarro G., Ibbadu G.F., Marra R., Rotiroti D., Loiacono A., Di Paola E.D., Russo E. Seizure susceptibility to various convulsant stimuli in dystrophyn-deficient mdx mice. Neuroscience Reasearch 50. 2004. 37-44

5. Goodwin F., Muntoni F., Dubowitz V. Epilepsy in Duchenne and Becker muscular dystrophies Eur J Paediatr Neurol. 1997. 115-119

6. Etemadifar M., Molaei S. Epilepsy in boys with Duchenne muscular dystrophy. J Res Med Sci. 2004. 116-119

7. Pane M., Messina S., Bruno C., D’Amico A., Villanova M., Brancalion B., Sivo S., Bianco F., Striano P., Battaglia D., Lettori D., Vita G.L., Bertini E., Gualandi F., Ricotti V., Ferlini A., Mercuri E. Duchenne muscular dystrophy and epilepsy. Neuromuscul. Disord. 2013. 23, 313-315

8. Hendriksen R.G.F., Hoogland G., Schipper S., Hendriksen J.G.M., Vles J.S.H., Aalbers M.W. A possible role of dystrophin in neuronal excitability: A review of the current literature. Neurosci Biobehav rev 51.2015 Apr;51:255-62

9. Ahn A.H., Kunkel L.M. The structural and functional diversity of dystrophin. Nat Genet. 1993 Apr;3(4):283-91

10. Ghedini P.C., Avellar M.C., De Lima T.C., Lima-Landman M.T., Lapa A.J., Souccar C. Quantitative changes of nicotinic receptors in the hippocampus of sytrophin-deficient mice. Brain Res. 2012 Nov;1483:96-104

11. Anderson, J.L., Head, S.I., Rae, C., Morley, J.W. Brain function in Duchenne muscular dystrophy. Brain: J. Neurol. 2002. 125, 4-13

12. Cyrulnik S.E., Hinton V. J. Duchenne muscular dystrophy: a cerebellar disorder? Neurosci. Biobehav. 2008. 
32:486-96

13. Lidov H.G. Dystrophin in the nervous system. Brain Pathol. 1996. 6, 63-77

14. Sekiguchi M., Zushida K., Yoshida M., Maekawa M., Kamichi S., Yoshida M., Sahara Y., Yuasa S., Takeda S., Wada K. A deficit of brain dystrophin impairs specific amygdala GABAergic transmission and enhances defensive behaviour in mice. Brain. 2009 Jan;132(Pt1):124-35

15. Knuesel, I., Mastrocola, M., Zuellig, R.A., Bornhauser, B., Schaub, M.C., Fritschy, J.M. Short communication: altered synaptic clustering of GABAA receptors in mice lacking dystrophin (mdx mice). Eur. J. Neurosci. 1999. 11, 4457-4462

16. Brünig, I., Suter, A., Knuesel, I., Lüscher, B., Fritschy, J.M. GABAergic terminals are required for postsynaptic clustering of dystrophin but not of GABA(A) receptors and gephyrin. J. Neurosci: Off. J. Soc. Neurosci. 2002 $22,4805-4813$

17. Anderson, J.L., Head, S.I., Morley, J.W. Duchenne muscular dystrophy and brain function. In: Hedge, M., Ankala, A. (Eds.), Muscular Dystrophy. Intech, 2012. pp. 91-122.

18. Perronnet, C., Vaillend, C. Dystrophins, utrophins, and associated scaffolding complexes: role in mammalian brain and implications for therapeutic strategies. J. Biomed. Biotechnol. 2010, 849426

19. Waite, A., Brown, S.C., Blake, D.J. The dystrophin-glycoprotein complex in brain development and disease. Trends Neurosci. 2010. 35, 487-496.

20. Connors, N.C., Adams, M.E., Froehner, S.C., Kofuji, P. The potassium channel Kir4.1 associates with the dystrophin-glycoprotein complex via alpha-syntrophin in glia. J Biol Chem. 2004 Jul 2;279(27):28387-92

21. Knuesel, I., Zuellig, R.A., Schaub, M.C., Fritschy, J.M. Alterations in dystrophin and utrophin expression parallel the reorganization of GABAergic synapses in a mouse model of temporal lobe epilepsy. Eur. J. Neurosci. 2001. Mar;13(6): 1113-1124

22. Fujimoto T., Itoh K., Yaoi T., Fushiki S. Somatodendritic and excitatory postsynaptic distribution of neuron-type dystrophin isoform, Dp40, in hippocampal neurons. Biochem Biophys Res Commun. 2014 Sep;452(1);79-84

23. Shiwaku H., Yoshimura N., Tamura T., Sone M., Ogishima S., Watase K., Tagawa K., Okazawa H. Suppression of the novel ER protein Maxer by mutant ataxin-1 in Bergman glia contributes to non-cell autonomous toxicity. EMBO J. $2010 \mathrm{Jul} ; 29(14): 2446-60$

24. Yang Y., Tang Y., Xing Y., Zhao M., Bao X., Sun D., Tang X., Wu Y., Xu H., Fan X. Activation of liver X receptor is protective against ethanol-induced developmental impairment of Bergmann glia and Purkinje neurons in the mouse cerebellum. Mol Neurobiol. 2014 Feb;49(1):176-86

25. Lavezzi A.M., Corna M.F., Matturri L. Neuronal nuclear antigen (NeuN): A useful marker of neuronal immaturity in sudden unexplained perinatal death. J Neurol Sci. 2013. 329:45-50

26. Snow W.M., Fry M., Anderson J.E. Increased density of dystrophin protein in the lateral versus the vermal mouse cerebellum. Cell Mol Neurobiol. 2013 May;33(4):513-20 